\title{
Curcuminoids for the management of hypertriglyceridaemia
}

Amirhossein Sahebkar

I read with great interest the Review by Watts et al. (Demystifying the management of hypertriglyceridaemia. Nat. Rev. Cardiol. 10, 648-661 [2013]), ${ }^{1}$ highlighting different strategies that are in development for treating hypertriglyceridaemia, including dual agonists of peroxisome proliferatoractivated receptors (PPARs), and inhibitors of cholesteryl ester transfer protein (CETP), microsomal triglyceride transfer protein (MTTP), diacylglycerol O-acyltransferase, angiopoietin-like protein, apoB, apoC-III, and proprotein convertase subtilisin kexin type 9. Although all these new agents have shown promise, the efficacy and safety of most of them is yet to be established owing to the lack of adequate clinical evidence. In this correspondence, I wish to discuss a multifunctional, natural product that should be listed among the emerging triglyceride-lowering agents, owing to its well-documented safety and efficacy in correcting hypertriglyceridaemia and associated comorbidities, including nonalcoholic fatty liver disease, acute pancreatitis, metabolic syndrome, obesity, and diabetes mellitus.

Curcuminoids are bioactive polyphenols responsible for the yellow colour-and most of the medicinal properties - of turmeric, a famous spice that has been commonly used for thousands of years in South Asian and Middle Eastern cuisine. Curcuminoids, comprising curcumin $(65-80 \%$ by weight), demethoxycurcumin (15-25\%), and bisdemethoxycurcumin (5-15\%), have been the subject of extensive research during the past 2 decades. They have been shown to possess numerous cardiometabolic benefits, owing to their ability to interact with diverse biological targets.

The triglyceride-lowering effects of curcuminoids have been confirmed in several experimental studies. More importantly, in randomized, controlled trials in healthy individuals, ${ }^{2}$ patients with obesity and dyslipidaemia, ${ }^{3}$ and patients with diabetes, ${ }^{4}$ curcuminoids have effectively lowered plasma triglyceride levels. The degree of lowering depends on the population studied as well as the curcuminoid dosage and formulation used. The reductions in plasma triglycerides by curcuminoids are comparable to those reported for new therapies in development, which were described by Watts et al. in their Review. ${ }^{1}$ In addition to their triglyceride-lowering effects, curcuminoids reduce hepatic fat accumulation and prevent steatosis by downregulating lipogenic factors (sterol regulatory element-binding protein-1, fatty acid synthase, acetyl-CoA carboxylase, acyl coenzyme A:cholesterol acyltransferase, 3-hydroxy-3-methylglutaryl -coenzyme A reductase, and CD36) ${ }^{5}$ and activating AMP-activated protein kinase signalling in liver. ${ }^{6}$ Curcuminoids also reduce biomarkers of systemic inflammation (including $\mathrm{C}$-reactive protein and NF- $\kappa B$-related cytokines), ${ }^{7,8}$ hepatocyte injury, and oxidative stress; ${ }^{2,9}$ improve insulin sensitivity and glycaemic control,4,10 increase plasma adiponectin; ${ }^{9}$ decrease plasma free fatty acid levels; ${ }^{4}$ activate PPAR- $\alpha$ and PPAR- $\gamma ;{ }^{6,11}$ and promote fatty acid $\beta$-oxidation and weight loss. ${ }^{6,10}$ All these effects are central to the prevention and treatment of nonalcoholic fatty liver disease, metabolic syndrome, and obesity. Interestingly, curcuminoids ameliorate both histopathologic and biochemical features of acute pancreatitis, ${ }^{12}$ a life-threatening condition that is causally related to severe hypertriglyceridaemia. Owing to their interaction with multiple targets, including PPAR- $\alpha$, PPAR- $\gamma,{ }^{6,11} \mathrm{CETP},{ }^{13}$ and lipoprotein lipase, ${ }^{4}$ curcuminoids are expected to affect both synthesis and catabolism of triglyceride-rich lipoproteins.

The triglyceride-lowering effects of curcuminoids should not be downplayed by the negative results of a published metaanalysis, ${ }^{14}$ as this meta-analysis was undertaken before the publication of recent randomized, controlled trials that clearly favour the substantial impact of curcuminoids on circulating triglyceride concentrations. ${ }^{2,4}$ Therefore, the exclusion of published studies that showed positive effects of curcuminoids could account for the negative result reported by the meta-analysis. Furthermore, the meta-analysis was based on data from a limited number of heterogeneous trials, in the majority of which curcuminoids were administered in the crude, and not the bioavailability-improved, formulations.

The use of curcuminoids has several potential advantages over some of the agents that are in the pipeline for treating hypertriglyceridaemia. First, curcuminoids have been repeatedly reported to be extremely safe, and daily doses as high as $8,000 \mathrm{mg}$ have been well tolerated. ${ }^{15}$ The concerns over hepatic triglyceride accumulation that occur with apoB antisense oligonucleotides and MTTP inhibitors, and off-target toxicities of dual PPAR $\alpha / \gamma$ agonists and CETP inhibitors, have not been observed in people taking curcuminoids, suggesting a wider indication for these natural products. Second, curcuminoids are orally active and widely and inexpensively available in purified forms, and the cost of their use is not comparable to those of biological therapies, such as monoclonal antibodies and antisense oligonucleotides. Finally, curcuminoid therapy is associated with several pleiotropic benefits for cardiovascular, cerebrovascular, and psychological well-being, as well as chemoprevention and tumour suppression. Low systemic bioavailability, which used to be regarded as the main obstacle to the efficacy of curcuminoids, has been satisfactorily addressed by using several formulation improvements, such as coadministration with absorption-enhancing adjuvants (for example, piperine and lecithin) and formulation into nanoparticles. ${ }^{16}$ All these products are currently available on the market. Given the efficacy and safety of curcuminoids, these phytochemicals are highly recommended to be administered for correcting hypertriglyceridaemia and other metabolic abnormalities in patients with obesity, metabolic syndrome, nonalcoholic fatty liver disease, or acute pancreatitis. 


\section{CORRESPONDENCE}

Biotechnology Research Center, Mashhad University of Medical Sciences, Mashhad 9177948564, Iran.

sahebkara@mums.ac.ir

\section{Competing interests}

The author declares no competing interests.

1. Watts, G. F., Ooi, E. M. \& Chan, D. C Demystifying the management of hypertriglyceridaemia. Nat. Rev. Cardiol. 10, 648-661 (2013).

2. Disilvestro, R. A., Joseph, E., Zhao, S. \& Bosmer, J. Diverse effects of a low dose supplement of lipidated curcumin in healthy middle aged people. Nutr. J. 11, 79 (2012).

3. Mohammadi, A et al. Effects of supplementation with curcuminoids on dyslipidemia in obese patients: a randomized crossover trial. Phytother. Res. 27, 374-379 (2013)

4. Na, L. X. et al. Curcuminoids exert glucose lowering effect in type 2 diabetes by decreasing serum free fatty acids: a double-blind, placebocontrolled trial. Mol. Nutr. Food Res. 57, 1569-1577 (2013).

5. Shin, S. K., Ha, T. Y., McGregor, R. A. \& Choi, M. S. Long-term curcumin administration protects against atherosclerosis via hepatic regulation of lipoprotein cholesterol metabolism. Mol. Nutr. Food Res. 55 1829-1840 (2011).

6. Um, M. Y., Hwang, K. H., Ahn, J. \& Ha, T. Y. Curcumin attenuates diet-induced hepatic steatosis by activating AMP-activated protein kinase. Basic Clin. Pharmacol. Toxicol. 113 152-157 (2013).

7. Sahebkar, A. Are curcuminoids effective C-reactive protein-lowering agents in clinical practice? Evidence from a meta-analysis. Phytother. Res. http://dx.doi.org/10.1002/ ptr.5045.

8. Soetikno, V. et al. Curcumin ameliorates macrophage infiltration by inhibiting NF- $\mathrm{KB}$ activation and proinflammatory cytokines in streptozotocin induced-diabetic nephropathy. Nutr. Metab. (Lond.) 8, 35 (2011).

9. Panahi, Y., Sahebkar, A., Parvin, S. \& Saadat, A A randomized controlled trial on the antiinflammatory effects of curcumin in patients with chronic sulphur mustard-induced cutaneous complications. Ann. Clin. Biochem. 49, 580-588 (2012).

10. Chuengsamarn, S., Rattanamongkolgul, S., Luechapudiporn, R., Phisalaphong, C. \& Jirawatnotai, S. Curcumin extract for prevention of type 2 diabetes. Diabetes Care 35, 2121-2127 (2012).
11. Nishiyama, T. et al. Curcuminoids and sesquiterpenoids in turmeric (Curcuma longa L.) suppress an increase in blood glucose level in type 2 diabetic KK-Ay mice. J. Agric. Food Chem. 53, 959-963 (2005).

12. Gulcubuk, A. et al. Effects of curcumin on proinflammatory cytokines and tissue injury in the early and late phases of experimental acute pancreatitis. Pancreatology 13, 347-354 (2013).

13. Shin, S. K., Ha, T. Y., McGregor, R. A. \& Choi, M. S. Long-term curcumin administration protects against atherosclerosis via hepatic regulation of lipoprotein cholesterol metabolism. Mol. Nutr. Food Res. 55 1829-1840 (2011)

14. Sahebkar, A. Systematic review and metaanalysis of randomized controlled trials investigating the effects of curcumin on blood lipid levels. Clin. Nutr. http://dx.doi.org/ 10.1016/j.clnu.2013.09.012.

15. Cheng, A. L. et al. Phase I clinical trial of curcumin, a chemopreventive agent, in patients with high-risk or pre-malignant lesions. Anticancer Res. 21, 2895-2900 (2001).

16. Bisht, S. \& Maitra, A. Systemic delivery of curcumin: 21st century solutions for an ancient conundrum. Curr. Drug Discov. Technol. 6 192-199 (2009). 